\title{
The Impact of Oxidative Stress on Adipose Tissue Energy Balance
}

\author{
Peter M. Masschelin ${ }^{1,2}$, Aaron R. Cox ${ }^{1}$, Natasha Chernis ${ }^{1}$ and Sean M. Hartig ${ }^{1,2 *}$ \\ ' Division of Endocrinology, Diabetes, and Metabolism, Department of Medicine, Baylor College of Medicine, Houston, TX, \\ United States, ${ }^{2}$ Department of Molecular and Cellular Biology, Baylor College of Medicine, Houston, TX, United States
}

Overnutrition and sedentary activity reinforce the growing trend of worldwide obesity, insulin resistance, and type 2 diabetes. However, we have limited insight into how food intake generates sophisticated metabolic perturbations associated with obesity. Accumulation of mitochondrial oxidative stress contributes to the metabolic changes in obesity, but the mechanisms and significance are unclear. In white adipose tissue (WAT), mitochondrial oxidative stress, and the generation of reactive oxygen species (ROS) impact the endocrine and metabolic function of fat cells. The central role of mitochondria in nutrient handling suggests pharmacological targeting of pathological oxidative stress likely improves the metabolic profile of obesity. This review will summarize the critical pathogenic mechanisms of obesity-driven oxidative stress in WAT.

Keywords: adipocyte, metabolism and obesity, oxidative stress, mitochondria, mitochondrial disorders

OPEN ACCESS

Edited by:

Tongzhi Wu,

University of Adelaide, Australia

Reviewed by:

P. Trayhurn

University of Liverpool,

United Kingdom

Hyun Roh,

Indiana University, United States

*Correspondence:

Sean M. Hartig

hartig@bcm.edu

Specialty section:

This article was submitted to

Clinical and Translational Physiology,

a section of the journal

Frontiers in Physiology

Received: 15 January 2019

Accepted: 30 December 2019

Published: 22 January 2020

Citation:

Masschelin PM, Cox AR

Chernis N and Hartig SM (2020) The

Impact of Oxidative Stress on

Adipose Tissue Energy Balance.

Front. Physiol. 10:1638.

doi: 10.3389/fphys.2019.01638
White adipose tissue (WAT) is an endocrine organ that stores energy in the form of lipids and secretes hormones essential for insulin sensitivity and energy homeostasis. The fat cell interprets nutritional, hormonal, and sympathetic tone in the tissue microenvironment to store and liberate fuels until whole-body energy demands necessitate fatty acid liberation. Like other cells, mitochondria in adipocytes assimilate signals that reflect the energy status of the cell and produce the majority of ATP from macronutrients through cellular respiration and oxidative phosphorylation (OXPHOS).

Obesity engenders nutrient stress that stifles mitochondrial capacity to sustain ATP levels in response to energy demands (Bournat and Brown, 2010; Liesa and Shirihai, 2013). Elevated mitochondrial substrate load consequently increases electron transport chain (ETC) activity and reactive oxygen species (ROS) production. Obese individuals exhibit higher levels of oxidative stress in WAT, including elevated ROS levels and decreased antioxidant activity coupled with alterations in adipokines required for insulin sensitivity (Furukawa et al., 2004). Moreover, oxidative stress associates with intra-abdominal obesity and insulin resistance (Furukawa et al., 2004; Frohnert et al., 2011). These results indicate the oxidizing environment in WAT of obese individuals likely impacts fat cell function and energy balance. Numerous questions remain, including how gradients of ROS inside the cell impact signaling cascades and gene regulation.

\section{NUTRIENT IMBALANCE PROVOKES MITOCHONDRIAL ROS}

Oxidative stress represents a disturbance in the equilibrium of ROS production and antioxidant defenses (Figure 1). At the molecular level, ROS mainly emerge from the mitochondrial ETC (Starkov, 2008; Murphy, 2009). Electron transfer through the ETC generates superoxide anions as byproducts, with complex I and III representing primary sources of ROS. Under certain conditions, complex II and other cellular ROS sources can contribute to the overall pool. Superoxide is the primary ROS species that reacts with Fe-containing proteins to generate $\mathrm{H}_{2} \mathrm{O}_{2} \cdot \mathrm{H}_{2} \mathrm{O}_{2}$ accumulation in the cell contributes directly to the metabolic imbalance linking excessive nutrient stress and 


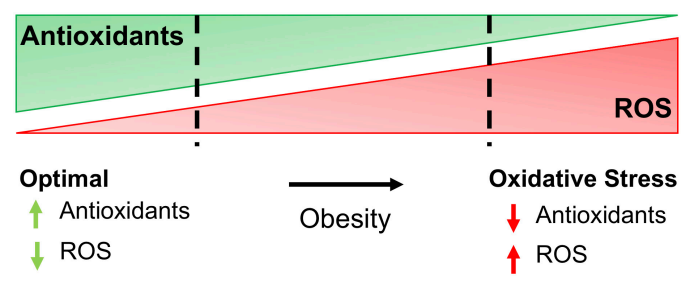

FIGURE 1 | The balance of antioxidants and ROS determine oxidative stress. For most cells, optimal redox conditions are achieved when higher levels of antioxidants are present to quench reactive oxygen species (ROS), maintaining ROS at low levels. Obesity and comorbidities increase ROS and decrease antioxidants in adipose, leading to oxidative stress and further complications of obesity, including insulin resistance and diabetes.

insulin resistance (Anderson et al., 2009; Akl et al., 2017; Fazakerley et al., 2018). However, ROS also encompass a diverse range of chemical entities, including nitric oxide, peroxynitrite, hypochlorous acid, singlet oxygen, and the hydroxyl radical. Consequently, the broad biological impacts of ROS derive from multiple cell and tissue microenvironments that divide physiological and pathological effects.

The conditions that favor mitochondrial superoxide production include reduction of electron carrier pools associated with the mitochondrial respiratory chain (NADH, flavins, ubiquinone), a high proton motive force, and elevated oxygen consumption within the mitochondria (Murphy, 2009). Overnutrition supplies excess electrons to the respiratory chain, while lack of physical activity and low ATP demand favors a high proton motive force with a low respiration rate, leading to mitochondrial superoxide formation and oxidative stress. By contrast, in mitochondria actively making ATP, superoxide production is low because the electron carriers are relatively oxidized, the proton motive force is small, and the respiration rate is high.

Prolonged oxidative stress directly impacts metabolism, including the activity of enzymes involved in the TCA cycle and the ETC (Quijano et al., 2016). The TCA cycle enzyme aconitase catalyzes the interconversion of citrate and isocitrate to regulate the availability of intermediates for lipid synthesis and ATP production. Citrate is the last common metabolite on the pathways for oxidation of acetyl-CoA and its export for fatty acid synthesis in the cytoplasm. Superoxide inhibits aconitase (Hausladen and Fridovich, 1994; Gardner et al., 1995), leading to diversion of acetyl-CoA away from OXPHOS and toward fat storage (Armstrong et al., 2004; Lushchak et al., 2014). This feedback loop may be part of an antioxidant defense mechanism that adapts prolonged mitochondrial superoxide production (Scandroglio et al., 2014). Acetyl-CoA diversion may slow delivery of electron carriers such as NADH to the respiratory chain, thereby decreasing ROS production (Armstrong et al., 2004).

Oxidative stress also impacts pyruvate dehydrogenase kinase 2 (PDK2) inhibition of the pyruvate dehydrogenase complex (PDC) (Hurd et al., 2012) and fat metabolism. ROS oxidize critical cysteine residues, disabling PDK2, and supporting
acetyl-CoA synthesis from glucose-derived pyruvate. Therefore, elevated mitochondrial superoxide and $\mathrm{H}_{2} \mathrm{O}_{2}$ couples PDC activity with aconitase interruption to divert citrate from the TCA cycle to the cytoplasm as triglycerides during overnutrition. These studies suggest persistent nutrient stress impairs the physiological behavior of crucial metabolic enzymes needed for balanced ATP generation and consumption.

\section{ANTIOXIDANT RESPONSE TO MITOCHONDRIAL ROS REGULATES ADIPOSE TISSUE FUNCTION}

A variety of peroxidases, including catalase, glutathione peroxidases, and peroxiredoxins (Prdxs) that control the levels of $\mathrm{H}_{2} \mathrm{O}_{2}$ in the cell and protect against ROS-induced damage by catalyzing the reduction of $\mathrm{H}_{2} \mathrm{O}_{2}$ into water. Along these lines, overexpression of catalase (Anderson et al., 2009; Barbosa et al., 2013; Lee et al., 2017; Paglialunga et al., 2017) or other methods that block $\mathrm{H}_{2} \mathrm{O}_{2}$ generation (Anderson et al., 2009; Boden et al., 2012) preserve insulin sensitivity in cell models and rodents fed high-fat diet.

The mitochondrial antioxidant peroxiredoxin $3(\operatorname{Prdx} 3)$ responds to oxidative stress and scavenges $\mathrm{H}_{2} \mathrm{O}_{2}$. Levels of Prdx3 are decreased in obese humans and mice, potentially contributing to oxidative stress intolerance (Huh et al., 2012). Whole-body deletion of $\operatorname{Pr} d x 3$ in mice causes obesity and increased expression of lipogenic genes in adipocytes, while decreasing expression of lipolytic genes. As a result, hypertrophic adipocytes exclusively accumulate excess lipids and cannot enable appropriate energy balance control. In addition to altering the balance of lipogenesis and lipolysis, Prdx3-deficient adipocytes exhibited increased superoxide production, decreased mitochondrial potential, and altered adipokine expression, including decreased adiponectin.

Okuno et al. (2018) created "Fat ROS-augmented" (AKO) and "Fat ROS-eliminated" (aP2-dTg) mice to address the question of how ROS affect WAT function. AKO mice leverage adipocytespecific ablation of glutamate-cysteine ligase $(G c l c)$ to disable the rate-limiting step in glutathione synthesis and increase ROS generation. AKO mice fed high fat/high sucrose (HF/HS) diet for 6 weeks had smaller adipocytes and decreased expression of lipogenic genes, including Acly, Scd1, Fasn, Acaca, and Srebf1. Insulin sensitivity was also reduced. Conversely, mice expressing rat catalase and human SOD1 under the aP2 promoter had the opposite phenotype. These mice (aP2-dTg) showed reduced $\mathrm{H}_{2} \mathrm{O}_{2}$ in subcutaneous and gonadal WAT. Feeding a HF/HS diet yielded beneficial subcutaneous and gonadal WAT expansion mirrored by increased expression of lipogenic genes (Acly, Scd1, Fasn, and Acaca) and insulin sensitivity.

While these data argue that increasing mitochondrial antioxidants protects against oxidative stress in WAT, genetic alteration of other mitochondrial antioxidants reveal different phenotypes. Manganese superoxide dismutase (MnSOD) is an important mitochondrial antioxidant that detoxifies superoxides (Holley et al., 2011). Adipocyte-specific knockout of MnSOD protected against diet-induced WAT expansion and weight gain (Han et al., 2016). Mechanistically, MnSOD knockout in 
adipocytes triggered an adaptive stress response that activated mitochondrial biogenesis and enhanced mitochondrial fatty acid oxidation, thereby preventing diet-induced obesity and insulin resistance. Increased ROS levels correlated with Uncoupling Protein 1 (UCP1) activation in subcutaneous WAT and higher energy expenditure (Han et al., 2016). These disparate features of mice that lack the $\operatorname{Prdx} 3$ and MnSOD genes coupled with therapeutic shortcomings of antioxidant therapies in human clinical trials (Fusco et al., 2007; Bjelakovic et al., 2013, 2014) suggest a more complex interaction of metabolism and redox balance in WAT.

\section{MITOCHONDRIAL REDOX REACTIONS GENERATE DIVERGENT INPUTS FOR CELLULAR SIGNALING}

The homeostatic systems that regulate oxidative stress in the lean state are largely repressed in obesity due to the accumulation of oxidized biomolecules within WAT. Excessive ROS irreversibly damages DNA, lipids, and proteins with adverse effects on cellular functions. Increased oxidative stress can alter proteins and lipids through direct and indirect pathways that culminate in oxidation of side chains and lipid-protein adduction (Grimsrud et al., 2008; Davies, 2016).

Reactive oxygen species oxidation of lipids ultimately generates lipid aldehydes that modify DNA, proteins, RNA, and other lipid species (Esterbauer et al., 1991; Uchida, 2003). Increased markers of lipid peroxidation, including thiobarbituric acid reactive substances (TBARS) and 8-epi-prostaglandin-F2 $\alpha$ (8-epi-PGF $2 \alpha$ ) are observed in individuals with higher BMI and waist circumference (Furukawa et al., 2004). Oxidized lipids and proteins preferentially accumulate in visceral depots compared to subcutaneous depots of obese mice (Long et al., 2013; Hauck et al., 2018, 2019) and humans (Frohnert et al., 2011), suggesting ROS modifications correlate with conditions associated with type 2 diabetes, including central fat accrual.

Highly reactive hydroxyl radicals $(\cdot \mathrm{OH})$ can be generated when excess $\mathrm{H}_{2} \mathrm{O}_{2}$ reacts with ferrous iron. Unlike $\mathrm{H}_{2} \mathrm{O}_{2}, \cdot \mathrm{OH}$ cannot undergo detoxification. Instead, $\cdot \mathrm{OH}$ removes electrons from neighboring lipids, proteins, and nucleic acids. Lipid aldehydes are highly electrophilic and prone to irreversible nucleophilic attack by the side chains of lysine (Lys), histidine (His), and cysteine (Cys) residues of proteins, resulting in a covalent lipid-protein adduct termed protein carbonylation (Schaur, 2003; Curtis et al., 2012). Furthermore, Lys, His, and Cys residues often cluster within active sites of enzymes or critical structural motifs, so their stable modification by lipids generally leads to inhibition or deactivation of protein function. However, recent work challenges the notion that ROSdriven modifications broadly degrade fat cell function. Brown adipose tissue (BAT) contains elevated levels of mitochondrial superoxide, mitochondrial $\mathrm{H}_{2} \mathrm{O}_{2}$, and oxidized lipids that correlate with acute activation of thermogenesis (Chouchani et al., 2016a,b). Mitochondrial ROS in BAT can converge on UCP1 C253 inducing cysteine sulfenylation (-SOH) (Chouchani et al., 2016a). Interestingly, UCP1 C253A does not disable thermogenic responses in brown adipocytes but desensitizes the protein to adrenergic activation of uncoupled respiration. Further exploration of physiological ROS signaling outputs and modifications may show how redox status in adipocytes contributes to energy balance.

Polyunsaturated fatty acids (PUFAs) are abundant in WAT and particularly sensitive to lipid peroxidation. One major consequence of lipid peroxidation is mitochondrial membrane damage (Kowaltowski and Vercesi, 1999). Also, peroxidation of PUFAs results in the release of diffusible reactive lipid aldehydes. Among the wide variety of reactive lipids formed through this mechanism, 4-hydroxy-non-enal (4-HNE) derived from oxidation of n6 fatty acids and 4-hydroxy-hexenal (4-HHE) from $\mathrm{n} 3$ fatty acid oxidation are the most widely studied in the context of adipose biology. The WAT of obese mice showed decreased metabolism of 4 -HNE, while stress response proteins, including glutathione-S-transferase M1, glutathione peroxidase 1, and Prdx (Grimsrud et al., 2007) were carbonylated. Lipid peroxidation end products can also inhibit insulin signaling as 4-HNE de-stabilizes IRS adapter proteins and insulin receptor $\beta$ (Demozay et al., 2008; Frohnert and Bernlohr, 2013).

Lipid peroxidation products also damage the function of transcription factors that contain zinc-finger motifs, histones, and other nuclear proteins of visceral fat cells isolated from obese mice (Hauck et al., 2018). The lipid peroxidation of transcriptional regulatory proteins presents a consolidated mechanism for retrograde ROS signaling from mitochondria to the nucleus. Although mitochondria are the most significant source of ROS, the discovery of lipid-protein adducts in the nucleus of adipocytes suggests either a different pool of ROS contributes to lipid peroxidation or a mechanism exists to sequester and shuttle reactive aldehydes to specific subcellular localizations (Hauck et al., 2018). As with ROS, the timing of protein carbonylation may be important for beneficial or pathologic effects. Acute carbonylation of substrates after exercise are potentially beneficial, while chronic accumulation of carbonylated proteins in the muscle and WAT of obese and sedentary individuals may be pathological and contribute to comorbidities of obesity (Frohnert and Bernlohr, 2013).

Additionally, ROS seem to be important in the cellular aspects of adipocyte differentiation. Numerous studies demonstrate that mitochondrial biogenesis increases during adipocyte differentiation (Wilson-Fritch et al., 2003; Lu et al., 2010; Zhang et al., 2013). Dramatic expansion of mitochondrial content enables higher metabolic rates to overcome the energetic demands of differentiation. Induction of differentiation correlates with superoxide generation from complex III, conversion of superoxide to $\mathrm{H}_{2} \mathrm{O}_{2}$, and activation of transcriptional machinery necessary for adipogenesis (Tormos et al., 2011). Mechanistically, ROS production in differentiating cells coincides with increased C/EBP $\beta$ binding to DNA and accelerated mitotic clonal expansion (Kim J.W. et al., 2007; Lee et al., 2009). However, obesity-mediated ROS induction also restricts mitochondrial biogenesis and adipocyte differentiation. Higher accumulation of 4-HNE adducts occurs in cultured differentiating preadipocytes from insulin-resistant compared to insulin-sensitive individuals. In this manner, treatment of 
primary subcutaneous preadipocytes from obese individuals with pathological levels of 4-HNE decreased markers associated with insulin sensitivity and mature fat cells (Dasuri et al., 2013; Elrayess et al., 2017). Other studies demonstrate that treatment with antioxidants decreases differentiation (Tormos et al., 2011) and disrupts UCP1-dependent thermogenic responses (Ro et al., 2014; Chouchani et al., 2016a). Divergent in vitro and in vivo findings illustrate existing challenges in defining the specifics of ROS signaling and its connectivity to metabolic diseases.

\section{OXIDATIVE STRESS CONTRIBUTES TO THE COMORBIDITIES OF METABOLIC DISEASES}

Nutrient overload has been linked to the development of insulin resistance. In a pioneering study, healthy men fed $\sim 6000 \mathrm{kcal} /$ day for 1 week exhibited WAT insulin resistance and oxidative stress in addition to protein oxidation and carbonylation (Boden et al., 2015). One carbonylated protein of importance was GLUT4, whose carbonylation likely impairs insulin-stimulated glucose uptake. Of note, systemic oxidative stress and insulin resistance did not coincide with inflammatory cytokines in plasma nor ER stress in WAT. These findings provide a causal link between oxidative stress and insulin resistance in humans.

Mitochondrial metabolism is often altered in inherited diseases, such as inborn errors of metabolism (IEMs) that impinge upon ROS generation. Inhibition of OXPHOS increases ROS generation due to a backlog of electrons in the various complexes, resulting in electron leak, ROS generation, and production of $\mathrm{H}_{2} \mathrm{O}_{2}$. In IEMs affecting the ETC or other pathways of ATP generation, increased oxidative stress is often observed, while the exact mechanisms for increased ROS production are unknown. It is hypothesized that mutations affecting the formation of the protein complexes in the ETC or mutations that modify their assembly increase ROS generation by facilitating electron leak (Olsen et al., 2015). Additionally, accumulation of toxic intermediates, often observed in IEMs, can increase the ROS generation by further decreasing OXPHOS activity, as in the case of medium-chain acyl-CoA dehydrogenase (MCAD) deficiency. MCAD deficiency reflects the accumulation of medium-chain fatty acid derivatives, including cis-4-decenoic acid, octanoate, and decanoate, with these metabolites altering levels of antioxidants and increasing markers of oxidative stress (Schuck et al., 2007, 2009). Intriguingly, IEMs display metabolic reprograming with a switch to glycolysis for both ATP production and muted ROS generation (Olsen et al., 2015). Specifically, in myoclonic epilepsy with ragged red fibers (MERRF), increased intracellular $\mathrm{H}_{2} \mathrm{O}_{2}$ levels correspond with increased AMPK phosphorylation and expression of GLUT1, hexokinase II, and lactate dehydrogenase. These results, as well as increased lactic acid production, all point to increased glycolysis (De la Mata et al., 2012; Wu and Wei, 2012). In multiple acylCoA dehydrogenase deficiency (MADD), mutations in ETFa, $E T F b$, or $E T F D H$, lead to decreased ATP production with an accumulation of organic acids, including glutaric acid as well as acyl-carnitines. A subset of these patients is riboflavin responsive (RR-MADD) with high dose riboflavin alleviating some symptoms. Similar to MERRF, many RR-MADD patients exhibit increased oxidative stress (Cornelius et al., 2013, 2014). This defect may be due to defective electron transfer and increased electron leak from the misfolded ETFDH protein and decreased binding of CoQ10 (Cornelius et al., 2013). Treatment with CoQ10, but not riboflavin, decreased ROS levels (Cornelius et al., 2013). Analysis of mitochondrial function from RR-MADD fibroblasts showed increased mitochondrial fragmentation and reduced $\beta$-oxidation, while supplementation with the antioxidant CoQ10 decreased fragmentation and mitophagy (Cornelius et al., 2014). While obesity and IEMs are distinct disorders, both conditions impinge on energy balance in WAT. Even though these disorders have very different manifestations, oxidative stress plays an important role in both and may be a therapeutic target. For example, CoQ10 is often given as a broad-spectrum treatment to individuals with IEMs, and while its effectiveness is debated, the anti-inflammatory effects may be beneficial in reducing oxidative stress and the pathogenesis of the disease (Cornelius et al., 2013; Acosta et al., 2016; Zhai et al., 2017).

\section{LEVERAGING REDOX BALANCE TO IMPROVE INSULIN SENSITIVITY}

Mitochondria represent control centers of many metabolic pathways. Interventions that enhance adipocyte mitochondrial function may also improve whole-body insulin sensitivity. Mitigation of mitochondrial ROS production and oxidative stress may be a possible therapeutic target in type 2 diabetes and IEMs because some mitochondrial-targeted antioxidants and other small molecule drugs improve metabolic profiles in mouse models (Feillet-Coudray et al., 2014; Fouret et al., 2015; Rivera-Barahona et al., 2017) and human studies (Escribano-Lopez et al., 2018).

Thiazolidinediones (TZDs) are PPAR $\gamma$ agonists used for treating type 2 diabetes (Kelly et al., 1999; King, 2000; Khan et al., 2002; Goldberg et al., 2005; Deeg et al., 2007). TZDs, such as rosiglitazone and pioglitazone, enhance insulin sensitivity by improving adipokine profiles (Maeda et al., 2001, 2002) and reducing fasting blood glucose levels (Boyle et al., 2002; Chappuis et al., 2007). TZDs also promote insulin sensitivity by directing fatty acids to subcutaneous fat, rather than visceral fat. Subcutaneous fat expandability, even in the context of obesity and type 2 diabetes, correlates with insulin sensitivity in rodents and humans (Ross et al., 1996; Miyazaki et al., 2002; Kim J.Y. et al., 2007; Tran et al., 2008; Porter et al., 2009). Numerous in vitro and in vivo studies demonstrate TZDs enhance mitochondrial biogenesis, content, function, and morphology. Rosiglitazone also induces cellular antioxidant enzymes responsible for the removal of ROS generated by increased mitochondrial activity in adipose tissue of diabetic rodents (Rong et al., 2007) and humans (Bogacka et al., 2005; Rong et al., 2007; Ahmed et al., 2010). It is now well established that anti-diabetic PPAR $\gamma$ agonists also activate a BAT gene program in white adipocytes, converting them to "beige" cells that express UCP1 (Tiraby et al., 2003; Wilson-Fritch et al., 2003; Bogacka et al., 2005; Ohno et al., 2005; Petrovic et al., 2010). Taken together, TZDs impact WAT 


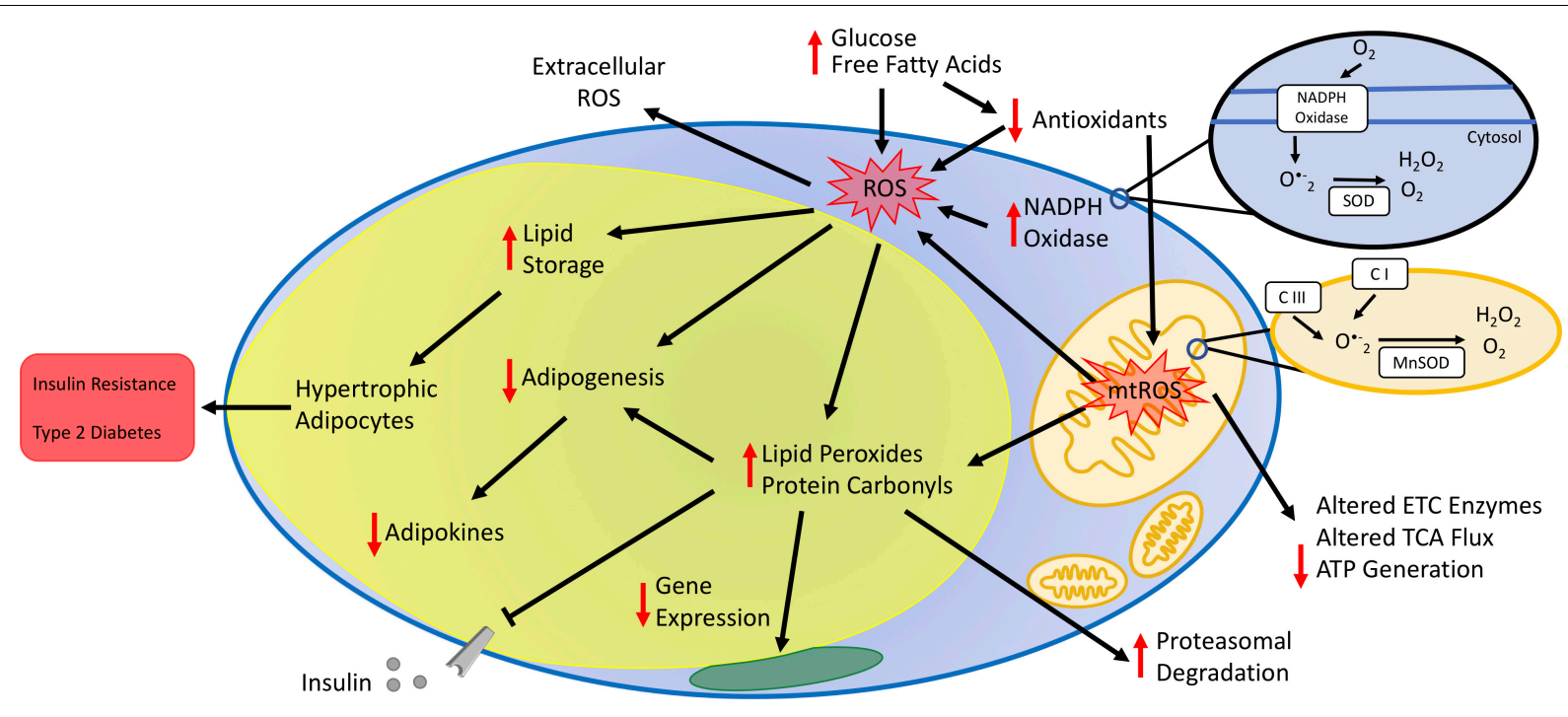

FIGURE 2 | Impact of oxidative stress on adipocyte function. Increased plasma glucose and free fatty acids contribute to increased oxidative stress by increasing the production of reactive oxygen species (ROS) and decreasing antioxidant concentrations. Increased oxidative stress occurs via enzymes in the cytoplasm, such as NADPH oxidase, and the mitochondria. The oxidative environment increases lipid storage resulting in hypertrophic adipocytes. Additionally, increased mitochondrial ROS (mtROS) alters the activity state of metabolic enzymes either directly or by changing the oxidative state of protein side-chains or by other post-translational modifications, including lipid peroxidation and protein carbonylation. Cumulatively, increased adipocyte oxidative stress decreases adipogenesis and secretion of adipokines, leading to unbalanced energy homeostasis, insulin resistance, and type 2 diabetes.

mitochondrial function in multiple ways that ultimately improve systemic fat metabolism and insulin sensitivity. Other therapeutic strategies include mitochondria-targeted scavengers (Smith et al., 2012) and chemical uncouplers that dissipate energy as heat (Perry et al., 2013; Goldgof et al., 2014). However, these methods to enhance mitochondrial function display a narrow therapeutic range that limits safe use for obesity.

Although the development of insulin resistance does not require impaired mitochondrial function (Hancock et al., 2008; Holloszy, 2013), pathways promoting insulin resistance may impair mitochondrial function and further increase ROS production, resulting in a detrimental feedback loop. Aerobic exercise and caloric restriction disrupt this vicious loop, potentially by preventing accumulation of injured mitochondrial proteins with substantial improvement of insulin sensitivity. In insulin-resistant people, aerobic exercise stimulates both mitochondrial biogenesis and efficiency concurrent with an enhancement of insulin action (Mul et al., 2015). Ultimately, exercise engages pathways that reduce ROS coupled with insulin sensitivity and improved mitochondrial function in WAT.

\section{CONCLUSION}

Obesity is the result of excessive expansion of WAT depots due to a chronic imbalance between energy intake and expenditure. Many studies demonstrate that oxidative stress in fat cells links obesity and its comorbidities. The fact that WAT remains the sole organ for storing surfeit lipid renders the macromolecules in adipocytes particularly vulnerable to carbonylation and other modifications driven by oxidative stress. Prolonged oxidative stress negatively influences endocrine and homeostatic performance of WAT, including disruption of hormone secretion, elevation of serum lipids, inadequate cellular antioxidant defenses, and impaired mitochondrial function (Figure 2). Metabolic challenges, such as persistent nutrient intake and sedentary behaviors that promote impaired glucose and lipid handling, also elevate mitochondrial ROS production to cause adipocyte dysfunction. Consequently, adipocytes cannot engage appropriate transcriptional and energetic responses to enable insulin sensitivity.

The increasing prevalence of obesity suggests lifestyle intervention as the principal method to treat obesity is unlikely to succeed. Currently, all available anti-obesity medications act by limiting energy intake through appetite suppression or inhibition of intestinal lipid absorption. However, these medications are largely ineffective and often have adverse side effects. The central role of mitochondria in nutrient handling provides a logical entry point for improving metabolism in obesity. While approaches to understanding and intervening in oxidative damage evolve, exploration of mitochondria redox balance may enable development of dietary and small molecule therapies for obesity and its comorbidities.

\section{AUTHOR CONTRIBUTIONS}

$\mathrm{PM}, \mathrm{AC}, \mathrm{NC}$, and $\mathrm{SH}$ wrote the manuscript. SH secured funding.

\section{FUNDING}

This work was funded by the American Diabetes Association \#1-18-IBS-105 and NIH R01DK114356. 


\section{REFERENCES}

Acosta, M. J., Vazquez Fonseca, L., Desbats, M. A., Cerqua, C., Zordan, R., Trevisson, E., et al. (2016). Coenzyme Q biosynthesis in health and disease. Biochim. Biophys. Acta 1857, 1079-1085. doi: 10.1016/j.bbabio.2016.03.036

Ahmed, M., Neville, M. J., Edelmann, M. J., Kessler, B. M., and Karpe, F. (2010). Proteomic analysis of human adipose tissue after rosiglitazone treatment shows coordinated changes to promote glucose uptake. Obesity 18, 27-34. doi: 10. 1038/oby.2009.208

Akl, M. G., Fawzy, E., Deif, M., Farouk, A., and Elshorbagy, A. K. (2017). Perturbed adipose tissue hydrogen peroxide metabolism in centrally obese men: association with insulin resistance. PLoS One 12:e0177268. doi: 10.1371/journal. pone. 0177268

Anderson, E. J., Lustig, M. E., Boyle, K. E., Woodlief, T. L., Kane, D. A., Lin, C. T., et al. (2009). Mitochondrial H2O2 emission and cellular redox state link excess fat intake to insulin resistance in both rodents and humans. J. Clin. Invest. 119, 573-581. doi: 10.1172/JCI37048

Armstrong, J. S., Whiteman, M., Yang, H., and Jones, D. P. (2004). The redox regulation of intermediary metabolism by a superoxide-aconitase rheostat. Bioessays 26, 894-900. doi: 10.1002/bies.20071

Barbosa, M. R., Sampaio, I. H., Teodoro, B. G., Sousa, T. A., Zoppi, C. C., and Queiroz, A. L. (2013). Hydrogen peroxide production regulates the mitochondrial function in insulin resistant muscle cells: effect of catalase overexpression. Biochim. Biophys. Acta 1832, 1591-1604. doi: 10.1016/j.bbadis. 2013.04.029

Bjelakovic, G., Nikolova, D., and Gluud, C. (2013). Antioxidant supplements to prevent mortality. JAMA 310, 1178-1179. doi: 10.1001/jama.2013.277028

Bjelakovic, G., Nikolova, D., and Gluud, C. (2014). Antioxidant supplements and mortality. Curr. Opin. Clin. Nutr. Metab. Care 17, 40-44. doi: 10.1097/MCO. 0000000000000009

Boden, G., Homko, C., Barrero, C. A., Stein, T. P., Chen, X., Cheung, P., et al. (2015). Excessive caloric intake acutely causes oxidative stress, GLUT4 carbonylation, and insulin resistance in healthy men. Sci. Transl. Med. 7:304re7. doi: 10.1126/scitranslmed.aac4765

Boden, M. J., Brandon, A. E., Tid-Ang, J. D., Preston, E., Wilks, D., Stuart, E., et al. (2012). Overexpression of manganese superoxide dismutase ameliorates high-fat diet-induced insulin resistance in rat skeletal muscle. Am. J. Physiol. Endocrinol. Metab. 303, E798-E805. doi: 10.1152/ajpendo.00577.2011

Bogacka, I., Ukropcova, B., McNeil, M., Gimble, J. M., and Smith, S. R. (2005). Structural and functional consequences of mitochondrial biogenesis in human adipocytes in vitro. J. Clin. Endocrinol. Metab. 90, 6650-6656. doi: 10.1210/jc. 2005-1024

Bournat, J. C., and Brown, C. W. (2010). Mitochondrial dysfunction in obesity. Curr. Opin. Endocrinol. Diabetes Obes. 17, 446-452. doi: 10.1097/med. 0b013e32833c3026

Boyle, P. J., King, A. B., Olansky, L., Marchetti, A., Lau, H., Magar, R., et al. (2002). Effects of pioglitazone and rosiglitazone on blood lipid levels and glycemic control in patients with type 2 diabetes mellitus: a retrospective review of randomly selected medical records. Clin. Ther. 24, 378-396. doi: 10.1016/ s0149-2918(02)85040-8

Chappuis, B., Braun, M., Stettler, C., Allemann, S., Diem, P., Lumb, P. J., et al. (2007). Differential effect of pioglitazone (PGZ) and rosiglitazone (RGZ) on postprandial glucose and lipid metabolism in patients with type 2 diabetes mellitus: a prospective, randomized crossover study. Diabetes Metab. Res. Rev. 23, 392-399. doi: 10.1002/dmrr.715

Chouchani, E. T., Kazak, L., Jedrychowski, M. P., Lu, G. Z., Erickson, B. K., Szpyt, J., et al. (2016a). Mitochondrial ROS regulate thermogenic energy expenditure and sulfenylation of UCP1. Nature 532, 112-116. doi: 10.1038/nature17399

Chouchani, E. T., Kazak, L., and Spiegelman, B. M. (2016b). Mitochondrial reactive oxygen species and adipose tissue thermogenesis: bridging physiology and mechanisms. J. Biol. Chem. 292, 16810-16816. doi: 10.1074/jbc.R117.789628

Cornelius, N., Byron, C., Hargreaves, I., Guerra, P. F., Furdek, A. K., Land, J., et al. (2013). Secondary coenzyme Q10 deficiency and oxidative stress in cultured fibroblasts from patients with riboflavin responsive multiple Acyl-CoA dehydrogenation deficiency. Hum. Mol. Genet. 22, 3819-3827. doi: 10.1093/ hmg/ddt 232

Cornelius, N., Corydon, T. J., Gregersen, N., and Olsen, R. K. (2014). Cellular consequences of oxidative stress in riboflavin responsive multiple acyl-CoA dehydrogenation deficiency patient fibroblasts. Hum. Mol. Genet. 23, 42854301. doi: $10.1093 / \mathrm{hmg} / \mathrm{ddu} 146$

Curtis, J. M., Hahn, W. S., Long, E. K., Burrill, J. S., Arriaga, E. A., and Bernlohr, D. A. (2012). Protein carbonylation and metabolic control systems. Trends Endocrinol. Metab. 23, 399-406. doi: 10.1016/j.tem.2012.05.008

Dasuri, K., Ebenezer, P., Fernandez-Kim, S. O., Zhang, L., Gao, Z., Bruce-Keller, A. J., et al. (2013). Role of physiological levels of 4-hydroxynonenal on adipocyte biology: implications for obesity and metabolic syndrome. Free Radic. Res. 47, 8-19. doi: 10.3109/10715762.2012.733003

Davies, M. J. (2016). Protein oxidation and peroxidation. Biochem. J. 473, 805-825. doi: 10.1042/BJ20151227

De la Mata, M., Garrido-Maraver, J., Cotan, D., Cordero, M. D., Oropesa-Avila, M., Izquierdo, L. G., et al. (2012). Recovery of MERRF fibroblasts and cybrids pathophysiology by coenzyme Q10. Neurotherapeutics 9, 446-463. doi: 10.1007/ s13311-012-0103-3

Deeg, M. A., Buse, J. B., Goldberg, R. B., Kendall, D. M., Zagar, A. J., Jacober, S. J., et al. (2007). Pioglitazone and rosiglitazone have different effects on serum lipoprotein particle concentrations and sizes in patients with type 2 diabetes and dyslipidemia. Diabetes Care 30, 2458-2464. doi: 10.2337/dc06-1903

Demozay, D., Mas, J. C., Rocchi, S., and Van Obberghen, E. (2008). FALDH reverses the deleterious action of oxidative stress induced by lipid peroxidation product 4-hydroxynonenal on insulin signaling in 3T3-L1 adipocytes. Diabetes Metab. Res. Rev. 57, 1216-1226. doi: 10.2337/db07-0389

Elrayess, M. A., Almuraikhy, S., Kafienah, W., Al-Menhali, A., Al-Khelaifi, F., Bashah, M., et al. (2017). 4-hydroxynonenal causes impairment of human subcutaneous adipogenesis and induction of adipocyte insulin resistance. Free Radic. Biol. Med. 104, 129-137. doi: 10.1016/j.freeradbiomed.2017. 01.015

Escribano-Lopez, I., Diaz-Morales, N., Iannantuoni, F., Lopez-Domenech, S., de Maranon, A. M., Abad-Jimenez, Z., et al. (2018). The mitochondrial antioxidant SS-31 increases SIRT1 levels and ameliorates inflammation, oxidative stress and leukocyte-endothelium interactions in type 2 diabetes. Sci. Rep. 8:15862. doi: 10.1038/s41598-018-34251-8

Esterbauer, H., Schaur, R. J., and Zollner, H. (1991). Chemistry and biochemistry of 4-hydroxynonenal, malonaldehyde and related aldehydes. Free Radic. Biol. Med. 11, 81-128. doi: 10.1016/0891-5849(91)90192-6

Fazakerley, D. J., Minard, A. Y., Krycer, J. R., Thomas, K. C., Stockli, J., Harney, D. J., et al. (2018). Mitochondrial oxidative stress causes insulin resistance without disrupting oxidative phosphorylation. J. Biol. Chem. 293, 7315-7328. doi: 10.1074/jbc.RA117.001254

Feillet-Coudray, C., Fouret, G., Ebabe Elle, R., Rieusset, J., Bonafos, B., Chabi, B., et al. (2014). The mitochondrial-targeted antioxidant MitoQ ameliorates metabolic syndrome features in obesogenic diet-fed rats better than Apocynin or Allopurinol. Free Radic. Res. 48, 1232-1246. doi: 10.3109/10715762.2014. 945079

Fouret, G., Tolika, E., Lecomte, J., Bonafos, B., Aoun, M., Murphy, M. P., et al. (2015). The mitochondrial-targeted antioxidant, MitoQ, increases liver mitochondrial cardiolipin content in obesogenic diet-fed rats. Biochim. Biophys. Acta 1847, 1025-1035. doi: 10.1016/j.bbabio.2015.05.019

Frohnert, B. I., and Bernlohr, D. A. (2013). Protein carbonylation, mitochondrial dysfunction, and insulin resistance. Adv. Nutr. 4, 157-163. doi: 10.3945/an.112. 003319

Frohnert, B. I., Sinaiko, A. R., Serrot, F. J., Foncea, R. E., Moran, A., and Ikramuddin, S. (2011). Increased adipose protein carbonylation in human obesity. Obesity 19, 1735-1741. doi: 10.1038/oby.2011.115

Furukawa, S., Fujita, T., Shimabukuro, M., Iwaki, M., Yamada, Y., and Nakajima, Y. (2004). Increased oxidative stress in obesity and its impact on metabolic syndrome. J. Clin. Invest. 114, 1752-1761.

Fusco, D., Colloca, G., Lo Monaco, M. R., and Cesari, M. (2007). Effects of antioxidant supplementation on the aging process. Clin. Interv. Aging 2, 377-387.

Gardner, P. R., Raineri, I., Epstein, L. B., and White, C. W. (1995). Superoxide radical and iron modulate aconitase activity in mammalian cells. J. Biol. Chem. 270, 13399-13405. doi: 10.1074/jbc.270.22.13399

Goldberg, R. B., Kendall, D. M., Deeg, M. A., Buse, J. B., Zagar, A. J., Pinaire, J. A., et al. (2005). A comparison of lipid and glycemic effects of pioglitazone and rosiglitazone in patients with type 2 diabetes and dyslipidemia. Diabetes Care 28, 1547-1554. doi: 10.2337/diacare.28.7.1547 
Goldgof, M., Xiao, C., Chanturiya, T., Jou, W., Gavrilova, O., and Reitman, M. L. (2014). The chemical uncoupler 2,4-dinitrophenol (DNP) protects against dietinduced obesity and improves energy homeostasis in mice at thermoneutrality. J. Biol. Chem. 289, 19341-19350. doi: 10.1074/jbc.M114.568204

Grimsrud, P. A., Picklo, M. J., Griffin, T. J., and Bernlohr, D. A. (2007). Carbonylation of adipose proteins in obesity and insulin resistance: identification of adipocyte fatty acid-binding protein as a cellular target of 4hydroxynonenal. Mol. Cell. Proteomics 6, 624-637. doi: 10.1074/mcp.m600120mcp200

Grimsrud, P. A., Xie, H., Griffin, T. J., and Bernlohr, D. A. (2008). Oxidative stress and covalent modification of protein with bioactive aldehydes. J. Biol. Chem. 283, 21837-21841. doi: 10.1074/jbc.R700019200

Han, Y. H., Buffolo, M., Pires, K. M., Pei, S., Scherer, P. E., and Boudina, S. (2016). Adipocyte-specific deletion of manganese superoxide dismutase protects from diet-induced obesity through increased mitochondrial uncoupling and biogenesis. Diabetes Metab. Res. Rev. 65, 2639-2651. doi: 10.2337/db160283

Hancock, C. R., Han, D. H., Chen, M., Terada, S., Yasuda, T., Wright, D. C., et al. (2008). High-fat diets cause insulin resistance despite an increase in muscle mitochondria. Proc. Natl. Acad. Sci. U.S.A. 105, 7815-7820. doi: 10.1073/pnas. 0802057105

Hauck, A. K., Huang, Y., Hertzel, A. V., and Bernlohr, D. A. (2019). Adipose oxidative stress and protein carbonylation. J. Biol. Chem. 294, 1083-1088. doi: 10.1074/jbc.R118.003214

Hauck, A. K., Zhou, T., Hahn, W., Petegrosso, R., Kuang, R., Chen, Y., et al. (2018). Obesity-induced protein carbonylation in murine adipose tissue regulates the DNA-binding domain of nuclear zinc finger proteins. J. Biol. Chem. 293, 13464-13476. doi: 10.1074/jbc.RA118.003469

Hausladen, A., and Fridovich, I. (1994). Superoxide and peroxynitrite inactivate aconitases, but nitric oxide does not. J. Biol. Chem. 269, 29405-29408.

Holley, A. K., Bakthavatchalu, V., Velez-Roman, J. M., and St Clair, D. K. (2011). Manganese superoxide dismutase: guardian of the powerhouse. Int. J. Mol. Sci. 12, 7114-7162. doi: 10.3390/ijms12107114

Holloszy, J. O. (2013). "Deficiency" of mitochondria in muscle does not cause insulin resistance. Diabetes Metab. Res. Rev. 62, 1036-1040. doi: 10.2337/db121107

Huh, J. Y., Kim, Y., Jeong, J., Park, J., Kim, I., Huh, K. H., et al. (2012). Peroxiredoxin 3 is a key molecule regulating adipocyte oxidative stress, mitochondrial biogenesis, and adipokine expression. Antioxid. Redox. Signal. 16, 229-243. doi: 10.1089/ars.2011.3952

Hurd, T. R., Collins, Y., Abakumova, I., Chouchani, E. T., Baranowski, B., Fearnley, I. M., et al. (2012). Inactivation of pyruvate dehydrogenase kinase 2 by mitochondrial reactive oxygen species. J. Biol. Chem. 287, 35153-35160. doi: 10.1074/jbc.M112.400002

Kelly, I. E., Han, T. S., Walsh, K., and Lean, M. E. (1999). Effects of a thiazolidinedione compound on body fat and fat distribution of patients with type 2 diabetes. Diabetes Care 22, 288-293. doi: 10.2337/diacare.22.2.288

Khan, M. A., St Peter, J. V., and Xue, J. L. (2002). A prospective, randomized comparison of the metabolic effects of pioglitazone or rosiglitazone in patients with type 2 diabetes who were previously treated with troglitazone. Diabetes Care 25, 708-711. doi: 10.2337/diacare.25.4.708

Kim, J. W., Tang, Q. Q., Li, X., and Lane, M. D. (2007). Effect of phosphorylation and S-S bond-induced dimerization on DNA binding and transcriptional activation by C/EBPbeta. Proc. Natl. Acad. Sci. U.S.A. 104, 1800-1804. doi: 10.1073/pnas.0611137104

Kim, J. Y., van de Wall, E., Laplante, M., Azzara, A., Trujillo, M. E., Hofmann, S. M., et al. (2007). Obesity-associated improvements in metabolic profile through expansion of adipose tissue. J. Clin. Invest. 117, 2621-2637. doi: 10.1172/ jci31021

King, A. B. (2000). A comparison in a clinical setting of the efficacy and side effects of three thiazolidinediones. Diabetes Care 23, 557. doi: 10.2337/diacare.23.4. $557 \mathrm{~b}$

Kowaltowski, A. J., and Vercesi, A. E. (1999). Mitochondrial damage induced by conditions of oxidative stress. Free Radic. Biol. Med. 26, 463-471. doi: 10.1016/ s0891-5849(98)00216-0

Lee, H., Lee, Y. J., Choi, H., Ko, E. H., and Kim, J. W. (2009). Reactive oxygen species facilitate adipocyte differentiation by accelerating mitotic clonal expansion. J. Biol. Chem. 284, 10601-10609. doi: 10.1074/jbc.M808742200
Lee, H. Y., Lee, J. S., Alves, T., Ladiges, W., Rabinovitch, P. S., Jurczak, M. J., et al. (2017). Mitochondrial-targeted catalase protects against high-fat diet-induced muscle insulin resistance by decreasing intramuscular lipid accumulation. Diabetes Metab. Res. Rev. 66, 2072-2081. doi: 10.2337/db16-1334

Liesa, M., and Shirihai, O. S. (2013). Mitochondrial dynamics in the regulation of nutrient utilization and energy expenditure. Cell Metab. 17, 491-506. doi: 10.1016/j.cmet.2013.03.002

Long, E. K., Olson, D. M., and Bernlohr, D. A. (2013). High-fat diet induces changes in adipose tissue trans-4-oxo-2-nonenal and trans-4-hydroxy-2-nonenal levels in a depot-specific manner. Free Radic. Biol. Med. 63, 390-398. doi: 10.1016/j. freeradbiomed.2013.05.030

Lu, R. H., Chouchani Ji, H., Chang, Z. G., Su, S. S., and Yang, G. S. (2010). Mitochondrial development and the influence of its dysfunction during rat adipocyte differentiation. Mol. Biol. Rep. 37, 2173-2182. doi: 10.1007/s11033009-9695-z

Lushchak, O. V., Piroddi, M., Galli, F., and Lushchak, V. I. (2014). Aconitase post-translational modification as a key in linkage between Krebs cycle, iron homeostasis, redox signaling, and metabolism of reactive oxygen species. Redox Rep. 19, 8-15. doi: 10.1179/1351000213Y.0000000073

Maeda, N., Shimomura, I., Kishida, K., Nishizawa, H., Matsuda, M., Nagaretani, H., et al. (2002). Diet-induced insulin resistance in mice lacking adiponectin/ACRP30. Nat. Med. 8, 731-737. doi: 10.1038/nm724

Maeda, N., Takahashi, M., Funahashi, T., Kihara, S., Nishizawa, H., Kishida, K., et al. (2001). PPARgamma ligands increase expression and plasma concentrations of adiponectin, an adipose-derived protein. Diabetes Metab. Res. Rev. 50, 2094-2099. doi: 10.2337/diabetes.50.9.2094

Miyazaki, Y., Mahankali, A., Matsuda, M., Mahankali, S., Hardies, J., Cusi, K., et al. (2002). Effect of pioglitazone on abdominal fat distribution and insulin sensitivity in type 2 diabetic patients. J. Clin. Endocrinol. Metab. 87, 2784-2791. doi: $10.1210 /$ jcem.87.6.8567

Mul, J. D., Stanford, K. I, Hirshman, M. F., and Goodyear, L. J. (2015). Exercise and regulation of carbohydrate metabolism. Prog. Mol. Biol. Transl. Sci. 135, 17-37. doi: 10.1016/bs.pmbts.2015.07.020

Murphy, M. P. (2009). How mitochondria produce reactive oxygen species. Biochem. J. 417, 1-13. doi: 10.1042/BJ20081386

Ohno, H., Shinoda, K., Spiegelman, B. M., and Kajimura, S. (2005). PPARgamma agonists induce a white-to-brown fat conversion through stabilization of PRDM16 protein. Cell Metab. 15, 395-404. doi: 10.1016/j.cmet.2012. 01.019

Okuno, Y., Fukuhara, A., Hashimoto, E., Kobayashi, H., Kobayashi, S., Otsuki, M., et al. (2018). Oxidative stress inhibits healthy adipose expansion through suppression of SREBF1-mediated lipogenic Pathway. Diabetes Metab. Res. Rev. 67, 1113-1127. doi: 10.2337/db17-1032

Olsen, R. K., Cornelius, N., and Gregersen, N. (2015). Redox signalling and mitochondrial stress responses; lessons from inborn errors of metabolism. J. Inherit. Metab. Dis. 38, 703-719. doi: 10.1007/s10545-015-9861-5

Paglialunga, S., Ludzki, A., Root-McCaig, J., and Holloway, G. P. (2017). In adipose tissue, increased mitochondrial emission of reactive oxygen species is important for short-term high-fat diet-induced insulin resistance in mice. Diabetologia 58 , 1071-1080. doi: 10.1007/s00125-015-3531-X

Perry, R. J., Kim, T., Zhang, X. M., Lee, H. Y., Pesta, D., Popov, V. B., et al. (2013). Reversal of hypertriglyceridemia, fatty liver disease, and insulin resistance by a liver-targeted mitochondrial uncoupler. Cell Metab. 18, 740-748. doi: 10.1016/ j.cmet.2013.10.004

Petrovic, N., Walden, T. B., Shabalina, I. G., Timmons, J. A., Cannon, B., and Nedergaard, J. (2010). Chronic peroxisome proliferator-activated receptor gamma (PPARgamma) activation of epididymally derived white adipocyte cultures reveals a population of thermogenically competent, UCP1-containing adipocytes molecularly distinct from classic brown adipocytes. J. Biol. Chem. 285, 7153-7164. doi: 10.1074/jbc.M109.053942

Porter, S. A., Massaro, J. M., Hoffmann, U., Vasan, R. S., O'Donnel, C. J., and Fox, C. S. (2009). Abdominal subcutaneous adipose tissue: a protective fat depot? Diabetes Care 32, 1068-1075. doi: 10.2337/dc08-2280

Quijano, C., Trujillo, M., Castro, L., and Trostchansky, A. (2016). Interplay between oxidant species and energy metabolism. Redox Biol. 8, 28-42. doi: 10.1016/j.redox.2015.11.010

Rivera-Barahona, A., Alonso-Barroso, E., Perez, B., Murphy, M. P., Richard, E., and Desviat, L. R. (2017). Treatment with antioxidants ameliorates oxidative 
damage in a mouse model of propionic acidemia. Mol. Genet. Metab. 122, 43-50. doi: 10.1016/j.ymgme.2017.07.009

Ro, S. H., Nam, M., Jang, I., Park, H. W., Park, H., Semple, I. A., et al. (2014). Sestrin 2 inhibits uncoupling protein 1 expression through suppressing reactive oxygen species. Proc. Natl. Acad. Sci. U.S.A. 111, 7849-7854. doi: 10.1073/pnas. 1401787111

Rong, J. X., Qiu, Y., Hansen, M. K., Zhu, L., Zhang, V., Xie, M., et al. (2007). Adipose mitochondrial biogenesis is suppressed in $\mathrm{db} / \mathrm{db}$ and high-fat diet-fed mice and improved by rosiglitazone. Diabetes Metab. Res. Rev. 56, 1751-1760. doi: $10.2337 / \mathrm{db} 06-1135$

Ross, R., Fortier, L., and Hudson, R. (1996). Separate associations between visceral and subcutaneous adipose tissue distribution, insulin and glucose levels in obese women. Diabetes Care 19, 1404-1411. doi: 10.2337/diacare.19.12. 1404

Scandroglio, F., Tortora, V., Radi, R., and Castro, L. (2014). Metabolic control analysis of mitochondrial aconitase: influence over respiration and mitochondrial superoxide and hydrogen peroxide production. Free Radic. Res. 48, 684-693. doi: 10.3109/10715762.2014.900175

Schaur, R. J. (2003). Basic aspects of the biochemical reactivity of 4hydroxynonenal. Mol. Aspects Med. 24, 149-159. doi: 10.1016/s0098-2997(03) 00009-8

Schuck, P. F., Ceolato, P. C., Ferreira, G. C., Tonin, A., Leipnitz, G., DutraFilho, C. S., et al. (2007). Oxidative stress induction by cis-4-decenoic acid: relevance for MCAD deficiency. Free Radic. Res. 41, 1261-1272. doi: 10.1080/ 10715760701687109

Schuck, P. F., Ferreira, G. C., Moura, A. P., Busanello, E. N., Tonin, A. M., Dutra-Filho, C. S., et al. (2009). Medium-chain fatty acids accumulating in MCAD deficiency elicit lipid and protein oxidative damage and decrease nonenzymatic antioxidant defenses in rat brain. Neurochem. Int. 54, 519-525. doi: 10.1016/j.neuint.2009.02.009

Smith, R. A., Hartley, R. C., Cocheme, H. M., and Murphy, M. P. (2012). Mitochondrial pharmacology. Trends Pharmacol. Sci. 33, 341-352. doi: 10. 1016/j.tips.2012.03.010

Starkov, A. A. (2008). The role of mitochondria in reactive oxygen species metabolism and signaling. Ann. N. Y. Acad. Sci. 1147, 37-52. doi: 10.1196/ annals. 1427.015
Tiraby, C., Tavernier, G., Lefort, C., Larrouy, D., Bouillaud, F., Ricquier, D., et al. (2003). Acquirement of brown fat cell features by human white adipocytes. J. Biol. Chem. 278, 33370-33376. doi: 10.1074/jbc.m305235200

Tormos, K. V., Anso, E., Hamanaka, R. B., Eisenbart, J., Joseph, J., Kalyanaraman, B., et al. (2011). Mitochondrial complex III ROS regulate adipocyte differentiation. Cell Metab. 14, 537-544. doi: 10.1016/j.cmet.2011.08.007

Tran, T. T., Yamamoto, Y., Gesta, S., and Kahn, C. R. (2008). Beneficial effects of subcutaneous fat transplantation on metabolism. Cell Metab. 7, 410-420. doi: 10.1016/j.cmet.2008.04.004

Uchida, K. (2003). 4-Hydroxy-2-nonenal: a product and mediator of oxidative stress. Prog. Lipid. Res. 42, 318-343. doi: 10.1016/s0163-7827(03)00014-6

Wilson-Fritch, L., Burkart, A., Bell, G., Mendelson, K., Leszyk, J., and Nicoloro, S. (2003). Mitochondrial biogenesis and remodeling during adipogenesis and in response to the insulin sensitizer rosiglitazone. Mol. Cell. Biol. 23, 1085-1094. doi: $10.1128 / \mathrm{mcb} .23 .3 .1085-1094.2003$

Wu, S. B., and Wei, Y. H. (2012). AMPK-mediated increase of glycolysis as an adaptive response to oxidative stress in human cells: implication of the cell survival in mitochondrial diseases. Biochim. Biophys. Acta 1822, 233-247. doi: 10.1016/j.bbadis.2011.09.014

Zhai, J., Bo, Y., Lu, Y., Liu, C., and Zhang, L. (2017). Effects of coenzyme Q10 on markers of inflammation: a systematic review and meta-analysis. PLoS One 12:e0170172. doi: 10.1371/journal.pone.0170172

Zhang, Y., Marsboom, G., Toth, P. T., and Rehman, J. (2013). Mitochondrial respiration regulates adipogenic differentiation of human mesenchymal stem cells. PLoS One 8:e77077. doi: 10.1371/journal.pone.0077077

Conflict of Interest: The authors declare that the research was conducted in the absence of any commercial or financial relationships that could be construed as a potential conflict of interest.

Copyright (C) 2020 Masschelin, Cox, Chernis and Hartig. This is an open-access article distributed under the terms of the Creative Commons Attribution License (CC BY). The use, distribution or reproduction in other forums is permitted, provided the original author(s) and the copyright owner(s) are credited and that the original publication in this journal is cited, in accordance with accepted academic practice. No use, distribution or reproduction is permitted which does not comply with these terms. 